\title{
Synthesis and Anticancer Evaluation of Some New Heterocyclic Scaffolds Incorporating the Acetanilide Moiety
}

\author{
Ehab Abdel-Latif*, Eman M. Keshk, Ali Saeed and Abdel-Galil M. Khalil \\ Department of Chemistry, Faculty of Science, Mansoura University, 35516 Mansoura, Egypt \\ Email: ehabattia00@gmx.net
}

\begin{abstract}
New heterocyclic scaffolds containing acetanilide moiety were synthesized as anticancer agents. The precursor $N$-(4-acetamidophenyl)-2-cyanoacetamide (3) was coupled smoothly with (un)substituted phenyl diazonium chlorides producing the hydrazones 4. Various 2-pyridones 6 were picked up through the treatment of 3 with different arylidene malononitriles. The precursor underwent reaction with phenyl isothiocyanate in $\mathrm{DMF} / \mathrm{KOH}$ followed by in situ addition of several $\alpha$-halogenated reagents afforded the corresponding thiazole derivatives 9 and 10 . Heating of thiocarbamoyl scaffold 11 with chloroacetone and phenacyl chloride resulted in the formation of 5substitutedthiophene-3-carboxamides 12. Condensation of the synthesized ketene N,S-acetal 13 with $\mathrm{NH}_{2} \mathrm{NH}_{2}$ furnished the corresponding pyrazole-4-carboxamide 14. The newly synthesized scaffolds were characterized by considering their spectral analyses and they evaluated for their in vitro anticancer activities to show promising results.
\end{abstract}
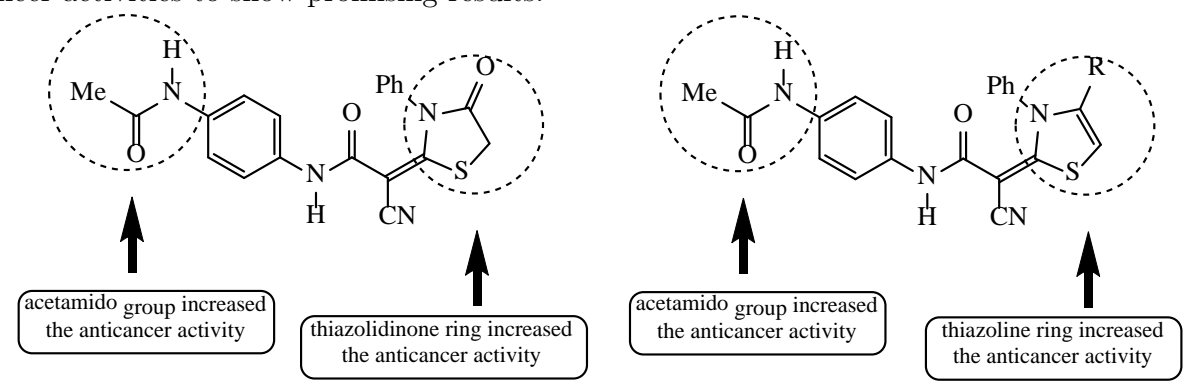

Keywords: 4-aminoacetanilide, hydrazones, 2-pyridones, thiazolidin-4-one, thiophene-3carboxamides, anticancer activity.

\section{$1 \quad$ Introduction}

Cyanoacetamide scaffolds have great interest in various branches of medicine chemistry because of their multiplicity uses in the drug industry. The nucleus of cyanoacetamide is important pharmacophore in many biologically active compounds and found in many clinically used drugs. Their pharmaceutical activities include: insulin releasing [1], anti-inflammatory [2], antifungal [3], carbonic anhydrase inhibitory [4], antimicrobial [5,6], anti-cancer and anti-tumor properties [7]. Among heterocyclic compounds: pyrazoles, thiophenes and thiazoles are intriguing group of scaffolds which have across the board pharmacological characteristics, for example, analgesic, antipyretic and anti-androgenic activities [8-11]. Pyrazoles also possess antidepressant, anti-inflammatory and anti-rheumatic activities [12-14]. In recent years, several thiazole derivatives were found as anticonvulsant activities [15-18], antioxidant activities [19], potential neuro protective agents [20,21] and anti-tumor agents [22,23]. Also, some pyridones have antibacterial [24], antitumor [25] and other interesting biological activities [26,27]. In addition, many number of thiophene derivatives have known to give pharmacological effect [28-30]. Furthermore, the chromene nucleus is so important in the chemistry of biological active agents [31] which has been used as antithrombotic, anti-inflammatory, antibacterial, cardio protectors, enzymatic inhibitors, antitumor and antifungal [32-36]. In view of these findings, the present work focuses on the synthesis of new heterocyclic scaffolds containing the acetanilide moiety and evaluates their anti-breast cancer activity. 


\section{Results and Discussion}

The important of aromatic amines activity is associated with the change probability of their - amino group to the cyanoacetamide group with furthermore function [23,24]. Cyanoacetylation of 4aminoacetanilide (1) with $N$-cyanoacetyl-pyrazole reagent 2 furnished the key start $N$-(4acetamidophenyl)-2-cyanoacetamide (3) (Scheme 1). Then, the activity of the methylene function of cyanoacetamide scaffold 3 towards reaction with diazonium chloride has been studied. Thus, cyanoacetamide scaffold 3 was diazocoupled with aryl diazonium chlorides, gotten from the suitable aromatic amines (aniline, 4-toluidine, and 4-chloroaniline) in pyridine to give the corresponding hydrazones $4 \mathrm{a}-\mathrm{c}$ (Scheme 1). The proposed structures of the latter reaction products are obtained according to spectral and analytical data.

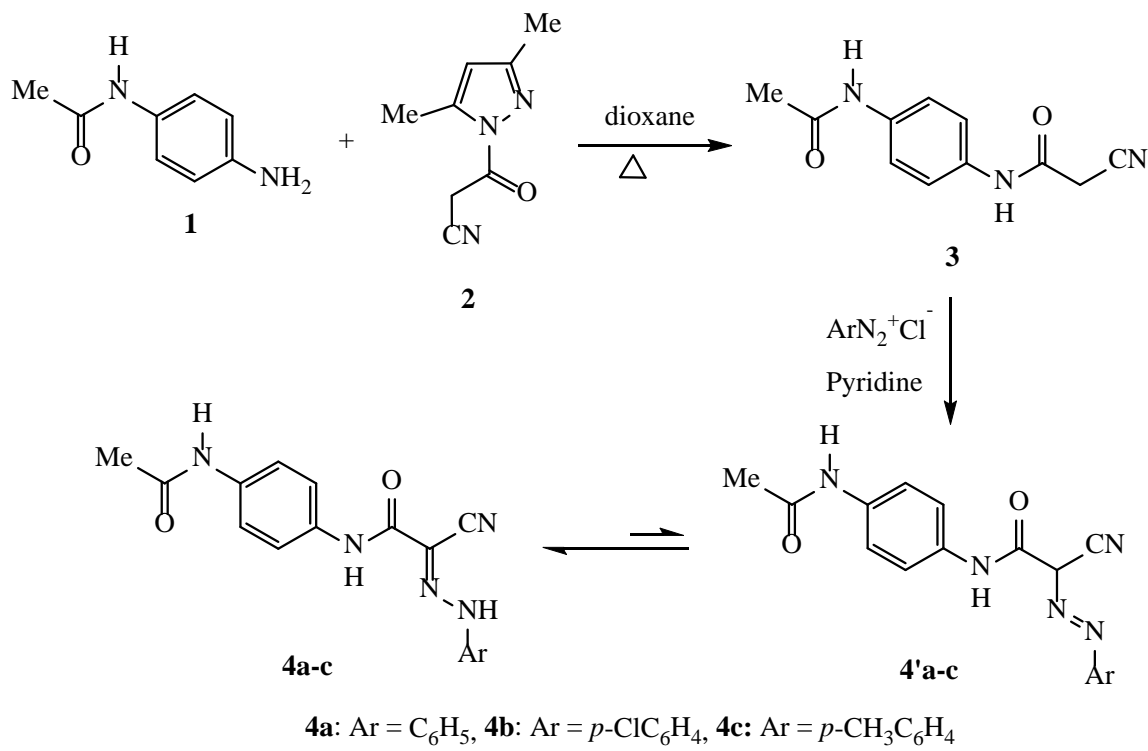

Scheme 1. Synthesis of N-(4-acetamidophenyl)-2-arylhydrazono-cyanoacetamides 4a-c.

In this manner, compound 3 was reacted with some aromatic aldehydes particularly benzaldehyde, 4anisaldehyde, 4-nitrobenzaldehyde, 4-chlorobenzaldehyde, 4-tolualdehyde, and 4(dimethylamino)benzaldehyde to afford the conformity unsaturated nitrile scaffolds 5a-f (Scheme 2). The IR of 5a, as an example of the synthesized scaffolds, clearly demonstrated absorptions at 3450, 3288, 2222 and $1649 \mathrm{~cm}^{-1}$ to indicate the presence of $\mathrm{NH}$, nitrile and carbonyl functional groups, respectively. ${ }^{1} \mathrm{H}$ NMR of the same scaffold demonstrated singlet for the protons of methyl function (2.03 ppm), multiplet for nine aromatic protons (7.54-7.74 ppm), singlet for olefinic proton (8.26 ppm) and two singlet signals for the protons of two NH functions (9.92 and $10.13 \mathrm{ppm})$. By heating in ethanol containing drops of piperidine as catalyst, pyridinone derivatives $6 \mathrm{a}-\mathrm{d}$ were obtained by the reaction of malononitrile with the synthesized arylidenes $5 \mathrm{a}-\mathrm{d}$. The chemical structure of these pyridinone scaffolds was secured by their alternative synthesis via the reaction of cyanoacetamide derivative 3 with arylidene-malononitrile derivatives in hot ethanol containing drops of piperidine. The resulted compounds $6 \mathrm{a}-\mathrm{d}$ were in perfect assent with the proposed structure according to elemental analyses and spectroscopic data.

In addition, cyclocondensation of cyanoacetanilide scaffold 3 with salicylaldehyde in ethanol containing drops of piperidine furnished $N$-(4-acetamidophenyl)-2-imino-2 $H$-chromene-3-carboxamide (7). According to IR spectrum of 7 , the absorptions at 3292,3261 and $1683 \mathrm{~cm}^{-1}$ indicated the presence of two $\mathrm{NH}$ and $\mathrm{C}=\mathrm{O}$ functions, respectively, while disappearance of any absorption band near $2200 \mathrm{~cm}^{-1}$ indicated the lack of nitrile function. The ${ }^{1} \mathrm{H}$ NMR spectrum of 7 revealed signals at 2.08 ppm (singlet for three protons, $\mathrm{CH}_{3}$ ), 7.21-7.81 ppm (multiplet for eight aromatic protons), $8.31 \mathrm{ppm}$ (singlet for the proton of chromene- $\mathrm{C}_{4}$ ) and $9.28,10.18$ and $13.11 \mathrm{ppm}$ for three protons of $\mathrm{NH}$ functions. 


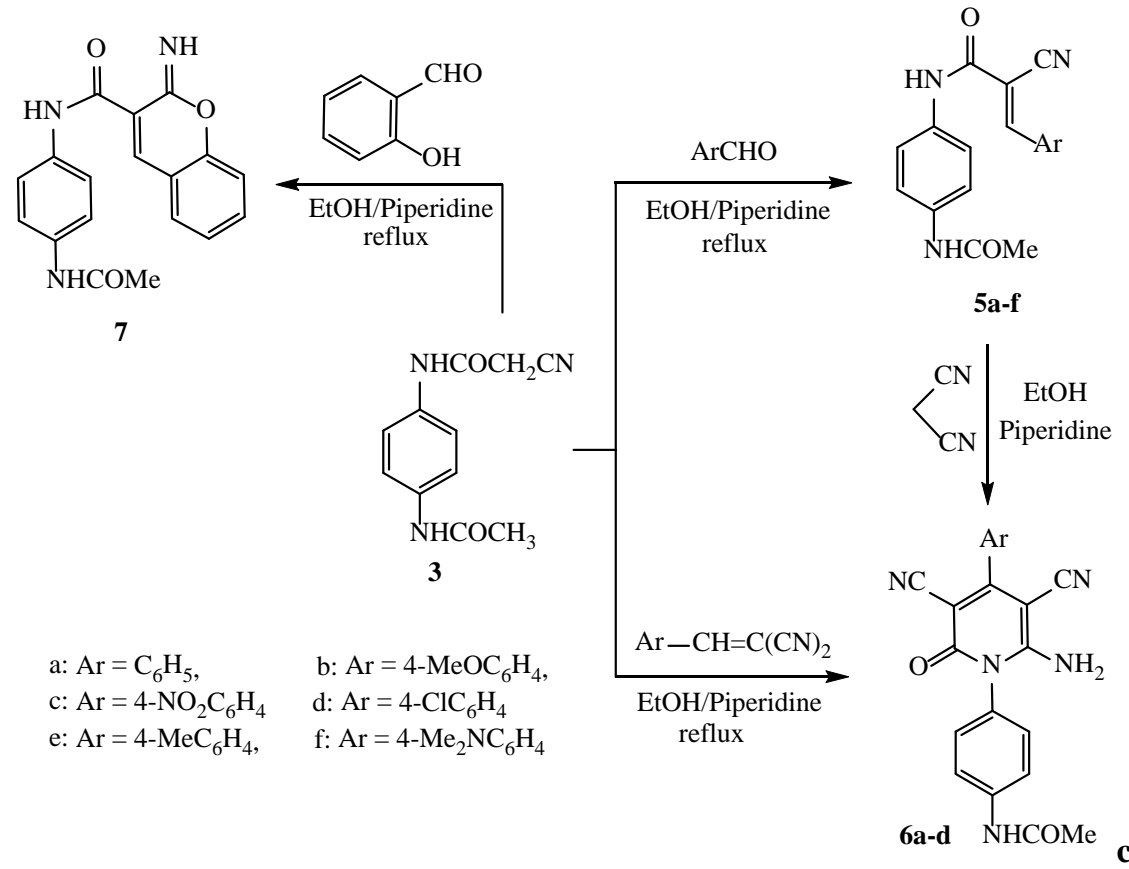

pyridones $6 \mathrm{a}-\mathrm{d}$ and imino-2H-chromene derivative 7.

cheme 2. Synthesis of

Treatment of cyanoacetamide scaffold 3 with phenyl isothiocyanate in dry DMF in the presence of an equimolar amount of $\mathrm{KOH}$ afforded the corresponding non-isolated sulphide salt 8, which underwent in situ stirring with ethyl chloroacetate, chloroacetone and phenacyl chloride furnished the corresponding thiazolidine-4-one, 4-methyl-3-phenylthiazoline and 3,4-diphenylthiazoline derivatives 9, 10a and 10b, respectively (Scheme 3). The absorption bands (3408, 3302, 2196 and $1743 \mathrm{~cm}^{-1}$ ) in the IR of thiazolidine-4-one scaffold 9 clearly indicated the presence of $\mathrm{NH}$, nitrile and cyclic carbonyl functions. The singlet signal for two protons at $3.99 \mathrm{ppm}$ in the ${ }^{1} \mathrm{H}$ NMR spectrum of 9 was the best telltale for the cyclic methylene group. The structure of thiazoline scaffold $10 \mathrm{a}$ was secured by ${ }^{1} \mathrm{H}$ NMR spectrum, which characterized by the presence of singlet at $6.90 \mathrm{ppm}$ for one olefinic proton (thiazole- $\mathrm{C}_{5}$ ).

The synthesis of $N$-(4-acetamidophenyl)-3-mercapto-acrylamide scaffold 11 was achieved by treating non-isolated potassium salt 8 with dilute $\mathrm{HCl}$. The IR spectrum of 11 clearly indicated the presence of $\mathrm{NH}$ (3457 and $\left.3318 \mathrm{~cm}^{-1}\right)$, nitrile $\left(2216 \mathrm{~cm}^{-1}\right)$ and carbonyl (broad $1665 \mathrm{~cm}^{-1}$ ) functional groups. Refluxing of thiocarbamoyl scaffold 11 with chloroacetone and/or phenacyl chloride in ethanol and triethylamine resulted in the formation of $\mathrm{N}$-(4-acetamidophenyl)-5-substitutedthiophene-3-carboxamides $12 \mathrm{a}$ and $12 \mathrm{~b}$, respectively. The IR spectra of $12 \mathrm{a}$ and $12 \mathrm{~b}$ were characterized by the presence of $\mathrm{NH}$ and $\mathrm{C}=\mathrm{O}$ absorption bands and the disappearance of nitrile absorption. In addition, ${ }^{1} \mathrm{H}$ NMR of $12 \mathrm{a}$ displayed four singlet signals at 2.01, 2.32, 11.55 and $11.88 \mathrm{ppm}$ due to the protons of two methyl and two NH functions, respectively.

Furthermore, in situ alkylation of non-isolable salt 8 was achieved by addition of methyl iodide to furnish $N$-(4-acetamidophenyl)-2-cyano-3-(methylthio)-3-(phenylamino)acrylamide (13). The reaction of 13 with hydrazine hydrate was proceeded in boiling ethanol to afford $N$-(4-acetamidophenyl)-5-amino-3(phenylamino)-1H-pyrazole-4-carboxamide (14) (Scheme 4). The IR spectrum indicated the lack of any absorption for the nitrile stretching and displayed absorption bands at 3445, 3261, 3176 and $1651 \mathrm{~cm}^{-1}$ due to $\mathrm{NH}_{2}, \mathrm{NH}$ and carbonyl groups, respectively. The ${ }^{1} \mathrm{H}$ NMR spectrum displayed singlet at $6.05 \mathrm{ppm}$ corresponding to $\mathrm{NH}_{2}$ protons, doublet and multiplet signals in the region $6.25-7.55 \mathrm{ppm}$ region due to aromatic protons, in addition to another three signals at $6.78,8.59$ and 8.78 ppm assignable to three NH protons. 


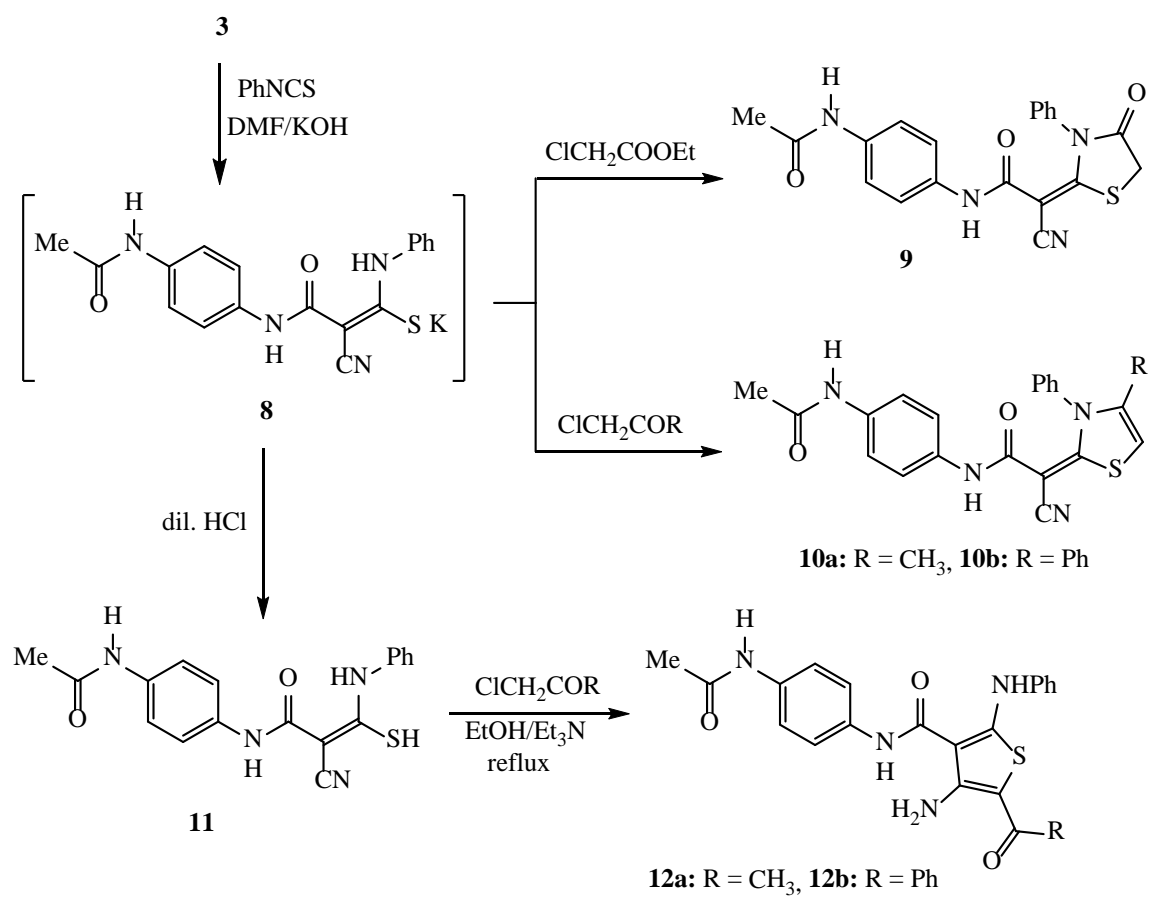

Scheme 3. Synthesis of thiazole and thiophene derivatives 9, 10 and 12 .

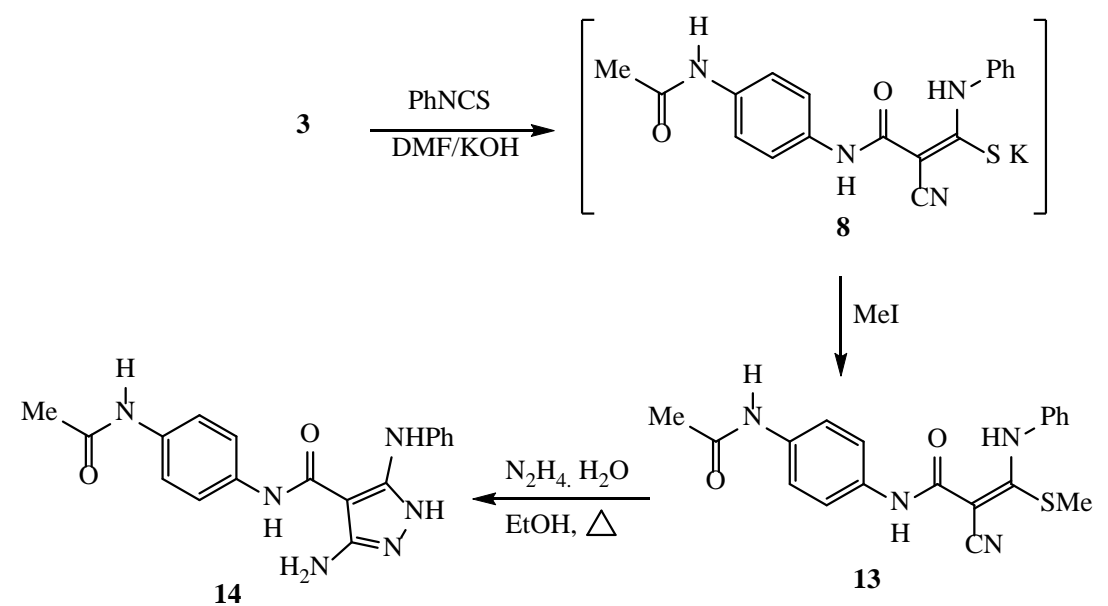

Scheme 4. Synthesis of pyrazole-4-carboxamide derivative 14 .

\section{$3 \quad$ In Vitro Anticancer Screening}

In vitro cytotoxic action of the synthesized various heterocyclic scaffolds was assessed against human breast cancer cell line, MCF7. 5-Fluorouracil, which is a standout amongst the best anticancer agents, was utilized as the reference sedate as a part of this work. The relationship between relative viability of cells $(\%)$ and concentration $(\mathrm{\mu g} / \mathrm{ml})$ was plotted to acquire the survival curve of breast cancer cell line (MCF7) (Figure 1). The results of statistical analysis of drug in vitro anticancer revealed a significant rapprochement between the control and tested compounds 4c, 5f, 9, 10a and 10b which proved to be the most active members in our study. They showed very strong potency towards MCF7 cancer cell line. 

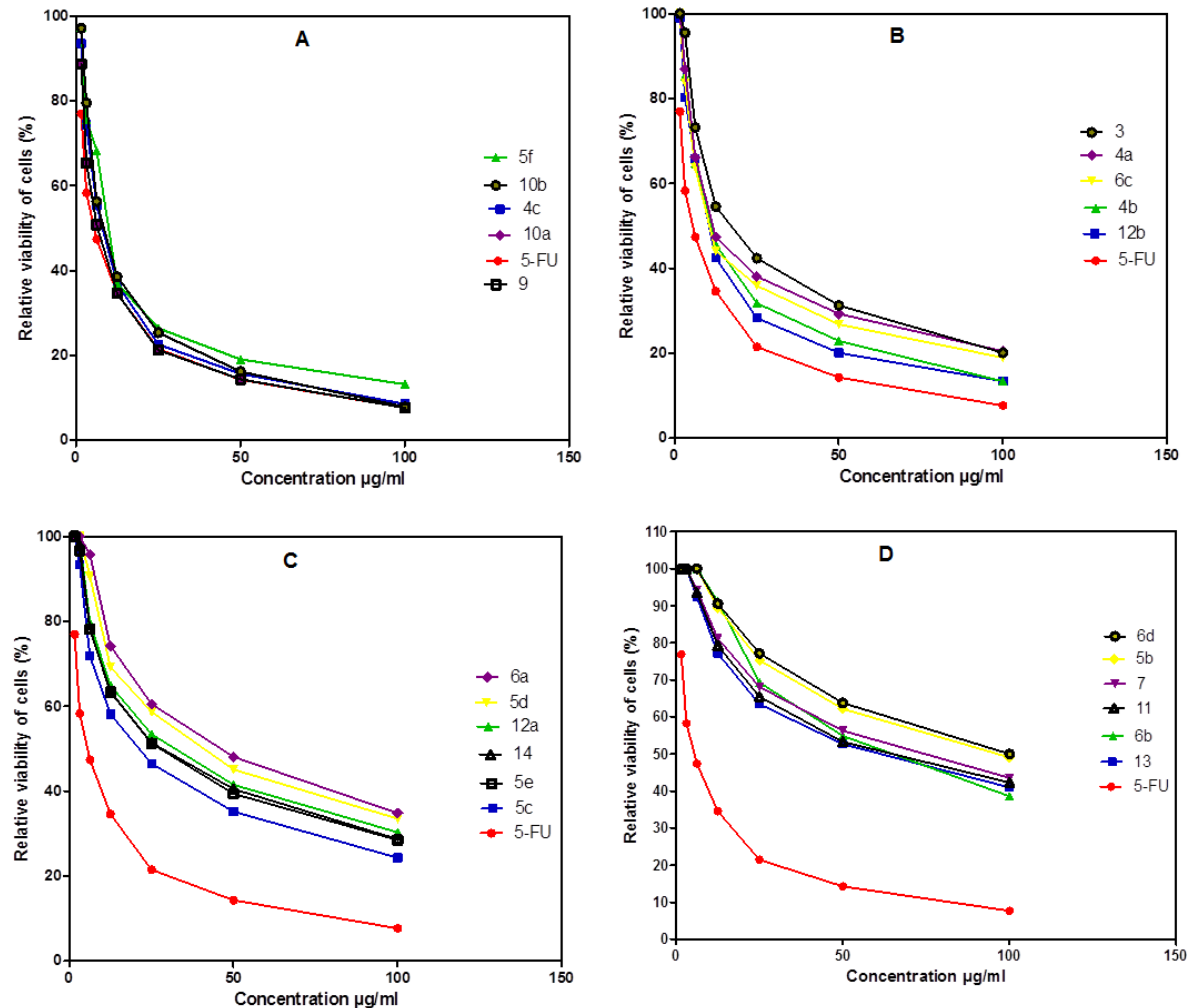

Figure 1. In vitro anticancer of relative viability of cells and drug concentration IC50 ( $\mathrm{\mu g} / \mathrm{ml})$ compared with the reference drug 5-FU (5-fluorouracil): A- (very strong), B- (strong), C- (moderate) and D- (weak cytotoxic).

In vitro cytotoxic activities of the synthesized scaffolds were recognized in Table 1. IC50 values refer to the concentration required for $100 \%$ inhibition of cell viability. Analysis of the data in Table 1 indicated that compounds 3, 4a-c, 5f, 6c, 9, 10a,b and 12b exhibited the lowest IC50, which means that they are the most effective cytotoxic drugs. Accordingly compounds 4c, 5f, 9, 10a and 10b can be used as very potent cytotoxic drug for breast carcinoma cell, while compounds $5 \mathrm{c}, 5 \mathrm{~d}, 6 \mathrm{a}, 6 \mathrm{f}$ and $12 \mathrm{a}$ have moderate IC50 which means that they are lower effective cytotoxic drug for breast carcinoma cell. On the other hand, the remaining compounds $5 \mathrm{~b}, 6 \mathrm{~b}, 6 \mathrm{~d}, 7$ and 13 are very weak cytotoxic drug compared with the reference drug, 5-fluorouracil.

Table 1. Cytotoxic activity of the synthesized scaffolds against human tumor cells.

\begin{tabular}{|c|c|}
\hline \multirow{2}{*}{ Compound } & In vitro Cytotoxicity IC50 $(\mathrm{\mu g} / \mathrm{ml})$ \\
\cline { 2 - 2 } & MCF-7 \\
\hline 5 -FU & $5.5 \pm 0.21$ \\
\hline 3 & $19.8 \pm 1.86$ \\
\hline $4 \mathrm{a}$ & $15.7 \pm 1.25$ \\
\hline 4b & $12.8 \pm 1.10$ \\
\hline $4 \mathrm{c}$ & $8.4 \pm 0.69$ \\
\hline $5 \mathrm{a}$ & $>100$ \\
\hline $5 \mathrm{~b}$ & $86.8 \pm 4.82$ \\
\hline $5 \mathrm{c}$ & $22.9 \pm 1.68$ \\
\hline $5 \mathrm{~d}$ & $40.8 \pm 2.94$ \\
\hline $5 \mathrm{e}$ & $29.4 \pm 2.06$ \\
\hline $5 \mathrm{f}$ & $10.4 \pm 0.88$ \\
\hline
\end{tabular}




\begin{tabular}{|c|c|}
\hline $6 \mathrm{a}$ & $46.0 \pm 3.75$ \\
\hline $6 \mathrm{~b}$ & $61.4 \pm 4.31$ \\
\hline $6 \mathrm{c}$ & $13.8 \pm 1.25$ \\
\hline $6 \mathrm{~d}$ & $91.3 \pm 5.69$ \\
\hline 7 & $67.3 \pm 4.82$ \\
\hline 9 & $5.3 \pm 0.28$ \\
\hline $10 \mathrm{a}$ & $6.9 \pm 0.41$ \\
\hline $10 \mathrm{~b}$ & $9.3 \pm 0.98$ \\
\hline 11 & $65.5 \pm 4.64$ \\
\hline $12 \mathrm{a}$ & $32.6 \pm 2.96$ \\
\hline $12 \mathrm{~b}$ & $11.4 \pm 1.03$ \\
\hline 13 & $57.2 \pm 3.87$ \\
\hline 14 & $30.4 \pm 1.92$ \\
\hline
\end{tabular}

IC50 ( $\mu \mathrm{g} / \mathrm{ml})$ : "1 - 10 (very strong); $11-20$ (strong); 21 - 50 (moderate); 51 - 100 (weak); above 100 (noncytotoxic); 5-FU = 5-fluorouracil"

The major findings gathered from the anticancer study of the unsaturated nitrile compounds 5a-f are highlighted as follow; the introduction of nitro and dimethylamino groups in compounds $5 \mathrm{c}$ and $5 \mathrm{f}$ provided higher anticancer activity than the other substituents (OMe, $\mathrm{Cl}$, and $\mathrm{Me}$ ). In addition, the newly synthesized thiazole scaffolds 9 and 10 exhibited similar or greater efficacy relative to the standard anticancer drug 5-fluorouracil. They could be important leads in continuing development against anticancer disease.

\section{$4 \quad$ Experimental}

\subsection{General}

All melting points (uncorrected) were measured on Gallenkamp electric melting point apparatus. Infrared spectra were determined on Mattson 5000 FTIR spectrometer ( $\mathrm{KBr}$ discs). The ${ }^{1} \mathrm{H}$ NMR spectra were recorded on a Varian XL $300 \mathrm{MHz}$ apparatus using DMSO- $d_{6}$ as a solvent. The mass spectra were acquired by EI mode at $70 \mathrm{eV}$ with Kratos MS equipment. Elemental analyses (C, H and N) were determined on Perkin-Elmer 2400 analyzer.

\subsection{Synthesis of N-(4-acetamidophenyl)-2-cyanoacetamide (3)}

A suspension of 4-aminoacetanilide $(1.50 \mathrm{~g}, 10 \mathrm{mmol})$ and $N$-cyanoacetyl-pyrazole reagent $2(1.63 \mathrm{~g}, 10$ mmol) was refluxed in dioxane $(20 \mathrm{~mL})$ for $4 \mathrm{~h}$. On cooling to room temperature, the resulting precipitate was filtered, dried and recrystallized from EtOH/DMF mixture (5:1) to afford the cyanoacetamide scaffold 3 .

Beige powder; yield (74\%); m.p. $269-270^{\circ} \mathrm{C}$. IR: 3289, $3172(\mathrm{NH}), 2256(\mathrm{C} \equiv \mathrm{N}), 1662(\mathrm{C}=\mathrm{O}) .{ }^{1} \mathrm{H}$ NMR $(\delta / \mathrm{ppm}): 2.02(\mathrm{~s}, 3 \mathrm{H}), 3.84(\mathrm{~s}, 2 \mathrm{H}), 7.44(\mathrm{~d}, 2 \mathrm{H}, \mathrm{J}=7.8 \mathrm{~Hz}), 7.51(\mathrm{~d}, 2 \mathrm{H}, \mathrm{J}=7.8 \mathrm{~Hz}), 9.86(\mathrm{~s}, 1 \mathrm{H})$, 10.18 (s, 1H). Anal. Calcd. for $\mathrm{C}_{11} \mathrm{H}_{11} \mathrm{~N}_{3} \mathrm{O}_{2}$ (217.23): C, 60.82; H, 5.10; N, 19.34\%. Found: C, 60.73; H, $5.12 ; \mathrm{N}, 19.22 \%$.

\subsection{Synthesis of N-(4-acetamidophenyl)-2-arylhydrazono-cyanoacetamides 4a-c}

A suspension of $5 \mathrm{mmol}$ aromatic amine (namely; aniline, 4-chloroaniline and 4-toluidine) in $1.5 \mathrm{~mL}$ conc. $\mathrm{HCl}$ was diazotized at $0-5^{\circ} \mathrm{C}$ with solution of $\mathrm{NaNO}_{2}(0.35 \mathrm{~g}, 5 \mathrm{mmol}$ in $10 \mathrm{~mL}$ water $)$. The freshly prepared diazonium chloride solution was added drop by drop to a suspension of $\mathrm{N}$-(4-acetamidophenyl)2-cyanoacetamide $(3)(1.08 \mathrm{~g}, 5 \mathrm{mmol})$ in pyridine $(20 \mathrm{~mL})$ at $0-5^{\circ} \mathrm{C}$. When the addition is completed, the stirring was continued for $2 \mathrm{~h}$ and the solid that formed was filtered and recrystallized from EtOH/DMF mixture (3:1).

$N$-(4-Acetamidophenyl)-2-(phenylhydrazono)-2-cyanoacetamide (4a): 
Red powder; yield (82\%); m.p. 245-246 ${ }^{\circ} \mathrm{C}$. IR: 3306, $3241(\mathrm{~N}-\mathrm{H}), 2211(\mathrm{C} \equiv \mathrm{N}), 1660(\mathrm{C}=\mathrm{O}) .{ }^{1} \mathrm{H}$ NMR ( $\delta / \mathrm{ppm}): 2.03(\mathrm{~s}, 3 \mathrm{H}), 7.22-7.65(\mathrm{~m}, 9 \mathrm{H}), 9.89(\mathrm{~s}, 1 \mathrm{H}), 10.17(\mathrm{~s}, 1 \mathrm{H}), 11.98$ (br. s, 1H). Anal. Calcd. for $\mathrm{C}_{17} \mathrm{H}_{15} \mathrm{~N}_{5} \mathrm{O}_{2}$ (321.34): C, 63.54; H, 4.71; N, 21.79\%. Found: C, 63.41; H, 4.63; N, 21.87\%.

$\mathrm{N}$-(4-Acetamidophenyl)-2-(4-chlorophenylhydrazono)-cyanoacetamide (4b):

Red powder; yield (70\%); m.p. 210-211 ${ }^{\circ} \mathrm{C}$. IR: 3351, 3221, $3188(\mathrm{NH}), 2208(\mathrm{C} \equiv \mathrm{N}), 1684,1665$ $(\mathrm{C}=\mathrm{O}) .{ }^{1} \mathrm{H} \operatorname{NMR}(\delta / \mathrm{ppm}): 2.04(\mathrm{~s}, 3 \mathrm{H}), 7.47(\mathrm{~d}, 2 \mathrm{H}, \mathrm{J}=9 \mathrm{~Hz}), 7.56-7.62(\mathrm{~m}, 4 \mathrm{H}), 7.92(\mathrm{~d}, 2 \mathrm{H}, \mathrm{J}=9$ $\mathrm{Hz}), 9.91(\mathrm{~s}, 1 \mathrm{H}), 10.22(\mathrm{~s}, 1 \mathrm{H}), 12.08(\mathrm{~s}, 1 \mathrm{H})$. Anal. Calcd. for $\mathrm{C}_{17} \mathrm{H}_{14} \mathrm{ClN}_{5} \mathrm{O}_{2}(355.78)$ : C, 57.39; H, 3.97; N, $19.68 \%$. Found: C, $57.30 ; \mathrm{H}, 3.93 ; \mathrm{N}, 19.57 \%$.

$\mathrm{N}$-(4-Acetamidophenyl)-2-(4-tolylhydrazono)-cyanoacetamide (4c)

Yellow crystals; yield (80\%); m.p. 255-256 ${ }^{\circ}$ C. IR: 3350, 3308, $3223(\mathrm{NH}), 2205(\mathrm{C} \equiv \mathrm{N}), 1681,1664$ $(\mathrm{C}=\mathrm{O}) .{ }^{1} \mathrm{H}$ NMR $(\delta / \mathrm{ppm}): 2.03(\mathrm{~s}, 3 \mathrm{H}), 2.29(\mathrm{~s}, 3 \mathrm{H}), 7.18-7.62(\mathrm{~m}, 8 \mathrm{H}), 9.81(\mathrm{~s}, 1 \mathrm{H}), 9.89(\mathrm{~s}, 1 \mathrm{H}), 11.78$ (s, 1H). MS (m/z, \%): $335\left(\mathrm{M}^{+}, 100.0\right), 293$ (10.2), 217 (14.6), 175 (19.6), 150 (74.2), 134 (21.3), 121 (11.4), 107 (63.6), 91 (26.3), 77 (16.9), 65 (7.1). Anal. Calcd. for $\mathrm{C}_{18} \mathrm{H}_{17} \mathrm{~N}_{5} \mathrm{O}_{2}$ (335.37): C, 64.47; H, 5.11; $\mathrm{N}, 20.88 \%$. Found: C, $64.59 ; \mathrm{H}, 5.04 ; \mathrm{N}, 20.78 \%$.

\subsection{Synthesis of $\mathrm{N}$-(4-acetamidophenyl)-3-aryl-2-cyano-acrylamides 5a-f}

A suspension of cyanoacetamide scaffold $3(1.08 \mathrm{~g}, 5 \mathrm{mmol})$ and the appropriate aromatic aldehyde (5 mmol) was heated under reflux for $3 \mathrm{~h}$ in EtOH $(25 \mathrm{ml})$ containing five drops of piperidine as catalyst. The resulting products (on cooling) were separated by filtration and then recrystallized from the suitable solvent to obtain $5 \mathrm{a}-\mathrm{f}$.

$\mathrm{N}$-(4-Acetamidophenyl)-2-cyano-3-phenylacrylamide (5a):

Yellow powder; yield (92\%); m.p. 245-246 ${ }^{\circ} \mathrm{C}(\mathrm{EtOH})$. IR: 3450, $3288(\mathrm{NH}), 2222(\mathrm{C} \equiv \mathrm{N}), 1649$ (broad, $\mathrm{C}=\mathrm{O}) .{ }^{1} \mathrm{H}$ NMR $(\delta / \mathrm{ppm}): 2.03(\mathrm{~s}, 3 \mathrm{H}), 7.54-7.74(\mathrm{~m}, 9 \mathrm{H}), 8.26(\mathrm{~s}, 1 \mathrm{H}), 9.92(\mathrm{~s}, 1 \mathrm{H}), 10.13(\mathrm{~s}, 1 \mathrm{H})$. Anal. Calcd. for $\mathrm{C}_{18} \mathrm{H}_{15} \mathrm{~N}_{3} \mathrm{O}_{2}$ (305.334): C, 70.81; H, 4.95; N, 13.76\%. Found: C, 70.94; H, 4.91; N, $13.83 \%$.

$\mathrm{N}$-(4-Acetamidophenyl)-2-cyano-3-(4-methoxyphenyl)acrylamide (5b):

Pale Yellow crystal; yield (89\%); m.p. 287-288 ${ }^{\circ} \mathrm{C}$ (EtOH/DMF mixture). IR: 3311, 3287 (NH), 2224 $(\mathrm{C} \equiv \mathrm{N}), 1675,1654(\mathrm{C}=\mathrm{O}) .{ }^{1} \mathrm{H}$ NMR $(\delta / \mathrm{ppm}): 2.03(\mathrm{~s}, 3 \mathrm{H}), 3.87(\mathrm{~s}, 3 \mathrm{H}), 7.17(\mathrm{~d}, 2 \mathrm{H}, \mathrm{J}=9 \mathrm{~Hz}), 7.56(\mathrm{~s}$, $4 \mathrm{H}), 8.02(\mathrm{~d}, 2 \mathrm{H}, \mathrm{J}=8.2 \mathrm{~Hz}), 8.18(\mathrm{~s}, 1 \mathrm{H}), 9.93(\mathrm{~s}, 1 \mathrm{H}), 10.21(\mathrm{~s}, 1 \mathrm{H})$. Anal. Calcd. for $\mathrm{C}_{19} \mathrm{H}_{17} \mathrm{~N}_{3} \mathrm{O}_{3}$ (335.36): C, 68.05; H, 5.11; N, 12.53\%. Found: C, 67.95; H, 5.01; N, $12.41 \%$.

$\mathrm{N}$-(4-Acetamidophenyl)-2-cyano-3-(4-nitrophenyl)acrylamide (5c):

Red powder; yield (74\%); m.p. 248-250 ${ }^{\circ} \mathrm{C}(\mathrm{EtOH} / \mathrm{DMF}$ mixture). IR: 3415, $3358(\mathrm{NH}), 2221(\mathrm{C} \equiv \mathrm{N})$, $1676(\mathrm{C}=\mathrm{O}) .{ }^{1} \mathrm{H}$ NMR $(\delta / \mathrm{ppm}): 2.03(\mathrm{~s}, 3 \mathrm{H}), 7.39(\mathrm{~d}, 2 \mathrm{H}, \mathrm{J}=9 \mathrm{~Hz}), 7.54-7.57(\mathrm{~m}, 4 \mathrm{H}), 8.11(\mathrm{~d}, 2 \mathrm{H}, \mathrm{J}$ $=8.2 \mathrm{~Hz}), 8.33(\mathrm{~s}, 1 \mathrm{H}), 9.92(\mathrm{~s}, 1 \mathrm{H}), 10.30(\mathrm{~s}, 1 \mathrm{H})$. Anal. Calcd. for $\mathrm{C}_{18} \mathrm{H}_{14} \mathrm{~N}_{4} \mathrm{O}_{4}(350.33): \mathrm{C}, 61.71 ; \mathrm{H}$, 4.03 ; N, 15.99\%. Found: C, $61.59 ; \mathrm{H}, 4.07 ; \mathrm{N}, 15.91 \%$.

$\mathrm{N}$-(4-Acetamidophenyl)-3-(4-chlorophenyl)-2-cyanoacrylamide (5d):

Yellow powder; yield (80\%); m.p. 288-290 ${ }^{\circ} \mathrm{C}(\mathrm{EtOH})$. IR: 3326, $3269(\mathrm{NH}), 2220(\mathrm{C} \equiv \mathrm{N}), 1673(\mathrm{C}=\mathrm{O})$. ${ }^{1} \mathrm{H}$ NMR $(\delta / \mathrm{ppm}): 2.03(\mathrm{~s}, 3 \mathrm{H}), 7.41(\mathrm{~d}, 2 \mathrm{H}, \mathrm{J}=9 \mathrm{~Hz}), 7.54-7.57(\mathrm{~m}, 4 \mathrm{H}), 8.20(\mathrm{~d}, 2 \mathrm{H}, \mathrm{J}=8.2 \mathrm{~Hz})$, $8.35(\mathrm{~s}, 1 \mathrm{H}), 9.95(\mathrm{~s}, 1 \mathrm{H}), 10.34(\mathrm{~s}, 1 \mathrm{H})$. MS (m/z, \%): $341\left(\mathrm{M}^{+}, 37.9\right), 339\left(\mathrm{M}^{+}, 100.0\right), 297(94.2), 190$ (10.3), 162 (8.5), 127 (12.6), 107 (94.7), 80 (50.8), 64 (20.3). Anal. Calcd. for $\mathrm{C}_{18} \mathrm{H}_{14} \mathrm{ClN}_{3} \mathrm{O}_{2}$ (339.78): C, $63.63 ; \mathrm{H}, 4.15 ; \mathrm{N}, 12.37 \%$. Found: C, 63.54; H, 4.21; N, $12.44 \%$.

$\mathrm{N}$-(4-Acetamidophenyl)-2-cyano-3-(4-tolyl)acrylamide (5e):

Yellow crystal, yield (89\%); m.p. 257-258 ${ }^{\circ} \mathrm{C}(\mathrm{EtOH})$. IR: 3324, $3282(\mathrm{NH}), 2220(\mathrm{C} \equiv \mathrm{N}), 1677,1656$ $(\mathrm{C}=\mathrm{O}) .{ }^{1} \mathrm{H} \operatorname{NMR}(\delta / \mathrm{ppm}): 2.03(\mathrm{~s}, 3 \mathrm{H}), 2.40(\mathrm{~s}, 3 \mathrm{H}), 7.40(\mathrm{~d}, 2 \mathrm{H}, \mathrm{J}=9 \mathrm{~Hz}), 7.57(\mathrm{~s}, 4 \mathrm{H}), 7.90(\mathrm{~d}, 2 \mathrm{H}, \mathrm{J}$ $=9 \mathrm{~Hz}), 8.20(\mathrm{~s}, 1 \mathrm{H}), 9.91(\mathrm{~s}, 1 \mathrm{H}), 10.26(\mathrm{~s}, 1 \mathrm{H})$. Anal. Calcd. for $\mathrm{C}_{19} \mathrm{H}_{17} \mathrm{~N}_{3} \mathrm{O}_{2}(319.36)$ : C, 71.46; H, 5.37; N, 13.16\%. Found: C, 71.53; H, 5.30; N, 13.07\%.

$\mathrm{N}$-(4-Acetamidophenyl)-2-cyano-3-(4-dimethylaminophenyl)acrylamide (5f):

Red powder; yield $(84 \%)$; m.p. $280-281^{\circ} \mathrm{C}(\mathrm{EtOH})$. IR: 3373, $3353(\mathrm{NH}), 2200(\mathrm{C} \equiv \mathrm{N}), 1687,1664$ $(\mathrm{C}=\mathrm{O}) .{ }^{1} \mathrm{H}$ NMR $(\delta / \mathrm{ppm}): 2.03(\mathrm{~s}, 3 \mathrm{H}), 3.07(\mathrm{~s}, 6 \mathrm{H}) 6.85(\mathrm{~d}, 2 \mathrm{H}, \mathrm{J}=9 \mathrm{~Hz}), 7.54(\mathrm{~s}, 4 \mathrm{H}), 7.90(\mathrm{~d}, 2 \mathrm{H}, \mathrm{J}$ $=9 \mathrm{~Hz}), 8.04(\mathrm{~s}, 1 \mathrm{H}), 9.88(\mathrm{~s}, 1 \mathrm{H}), 9.93(\mathrm{~s}, 1 \mathrm{H})$. Anal. Calcd. for $\mathrm{C}_{20} \mathrm{H}_{20} \mathrm{~N}_{4} \mathrm{O}_{2}(348.41): \mathrm{C}, 68.95 ; \mathrm{H}, 5.79$; N, 16.08\%. Found: C, 68.82; H, 5.86; N, 16.19\%. 


\subsection{Synthesis of 1-(4-acetamidophenyl)-6-amino-4-aryl-3,5-dicyano-2-oxopyridine derivatives $6 \mathrm{a}-\mathrm{d}$}

Method A: To a mixture of arylidene derivatives $5 \mathrm{a}-\mathrm{d}(5 \mathrm{mmol})$ and malononitrile $(0.33 \mathrm{~g}, 5 \mathrm{mmol})$ in $20 \mathrm{~mL}$ of ethanol, five drops of piperidine was added and then heated under reflux for $3 \mathrm{~h}$. On cooling to room temperature, the precipitated solid was filtered and recrystallized from EtOH/DMF mixture (3:1) to obtain the pyridine scaffolds $6 \mathrm{a}-\mathrm{d}$.

Method B: Equimolar weights of cyanoacetamide scaffold 3 (1.08 g, $5 \mathrm{mmol}$ ) and the appropriate (2arylidenemalononitrile derivative $(5 \mathrm{mmol})$ was refluxed for $3 \mathrm{~h}$ in $20 \mathrm{~mL}$ ethanol previously treated with drops of piperidine. The precipitate, which was found after cooling, was filtered and recrystallized from EtOH/DMF mixture (3:1).

1-(4-Acetamidophenyl)-6-amino-3,5-dicyano-2-oxo-4-phenylpyridine (6a):

Pale yellow powder; yield (70\%); m.p. $>300^{\circ} \mathrm{C}$. IR: 3451, 3373, $3191\left(\mathrm{NH}_{2}\right.$ and $\left.\mathrm{NH}\right), 2211(\mathrm{C} \equiv \mathrm{N})$, $1684(\mathrm{C}=\mathrm{O}) .{ }^{1} \mathrm{H} \operatorname{NMR}(\delta / \mathrm{ppm}): 2.01(\mathrm{~s}, 3 \mathrm{H}), 7.55-7.81(\mathrm{~m}, 11 \mathrm{H}), 10.22(\mathrm{~s}, 1 \mathrm{H})$. Anal. Calcd. for $\mathrm{C}_{21} \mathrm{H}_{15} \mathrm{~N}_{5} \mathrm{O}_{2}$ (369.38): C, 68.28; H, 4.09; N, 18.96\%. Found: C, 68.16; H, 4.15; N, $18.88 \%$.

1-(4-Acetamidophenyl)-6-amino-3,5-dicyano-4-(4-methoxyphenyl)-2-oxopyridine (6b):

Yellow crystal; yield (86\%); m.p. > 300 ${ }^{\circ}$. IR: 3446, 3305, $3196\left(\mathrm{NH}_{2}\right.$ and $\left.\mathrm{NH}\right), 2214(\mathrm{C} \equiv \mathrm{N}), 1681$, $1658(\mathrm{C}=\mathrm{O}) .{ }^{1} \mathrm{H}$ NMR $(\delta / \mathrm{ppm}): 2.02(\mathrm{~s}, 3 \mathrm{H}), 3.86(\mathrm{~s}, 3 \mathrm{H}), 7.11(\mathrm{~d}, 2 \mathrm{H}, \mathrm{J}=9 \mathrm{~Hz}), 7.26(\mathrm{~d}, 2 \mathrm{H}, \mathrm{J}=9$ $\mathrm{Hz}), 7.51$ (d, $2 \mathrm{H}, \mathrm{J}=9 \mathrm{~Hz}), 7.78(\mathrm{~d}, 4 \mathrm{H}), 10.16(\mathrm{~s}, 1 \mathrm{H})$. Anal. Calcd. for $\mathrm{C}_{22} \mathrm{H}_{17} \mathrm{~N}_{5} \mathrm{O}_{3}(399.41):$ C, 66.16; H, 4.29; N, 17.53\%. Found: C, 66.08; H, 4.27; N, 17.47\%.

1-(4-Acetamidophenyl)-6-amino-3,5-dicyano-4-(4-nitrophenyl)-2-oxopyridine (6c):

Brown powder; yield (54\%); m.p. > 300 ${ }^{\circ} \mathrm{C}$. IR: 3447, 3292, $3174\left(\mathrm{NH}_{2}\right.$ and $\left.\mathrm{NH}\right), 2257(\mathrm{C} \equiv \mathrm{N}), 1666$ $(\mathrm{C}=\mathrm{O}) .{ }^{1} \mathrm{H}$ NMR $(\delta / \mathrm{ppm}): 2.01(\mathrm{~s}, 3 \mathrm{H}), 7.36(\mathrm{~d}, 2 \mathrm{H}, \mathrm{J}=8.2 \mathrm{~Hz}), 7.45-7.62(\mathrm{~m}, 6 \mathrm{H}), 8.10(\mathrm{~d}, 2 \mathrm{H}, \mathrm{J}=9$ $\mathrm{Hz}), 10.28$ (s, 1H). Anal. Calcd. for $\mathrm{C}_{21} \mathrm{H}_{14} \mathrm{~N}_{6} \mathrm{O}_{4}$ (414.38): C, 60.87; H, 3.41; N, 20.28\%. Found: C, 60.63; $\mathrm{H}, 3.33 ; \mathrm{N}, 20.16 \%$.

1-(4-Acetamidophenyl)-6-amino-4-(4-chlorophenyl)-3,5-dicyano-2-oxopyridine (6d):

Pale yellow powder; yield $(75 \%)$; m.p. $>300^{\circ} \mathrm{C}$. IR: 3456, 3315, $3186\left(\mathrm{NH}_{2}\right.$ and $\left.\mathrm{NH}\right), 2217(\mathrm{C} \equiv \mathrm{N})$, 1682, $1661(\mathrm{C}=\mathrm{O}) .{ }^{1} \mathrm{H} \operatorname{NMR}(\delta / \mathrm{ppm}): 2.02(\mathrm{~s}, 3 \mathrm{H}), 7.39(\mathrm{~d}, 2 \mathrm{H}, \mathrm{J}=8.2 \mathrm{~Hz}), 7.46-7.61(\mathrm{~m}, 6 \mathrm{H}), 8.13(\mathrm{~d}$, $2 \mathrm{H}, \mathrm{J}=9 \mathrm{~Hz}), 10.31(\mathrm{~s}, 1 \mathrm{H})$. MS (m/z, \%): $405\left(\mathrm{M}^{+}, 2.8\right), 403\left(\mathrm{M}^{+}, 8.0\right), 361$ (14.4), 108 (7.6), 92 (5.6), 80 (17.4), 64 (100.0). Anal. Calcd. for $\mathrm{C}_{21} \mathrm{H}_{14} \mathrm{ClN}_{5} \mathrm{O}_{2}$ (403.83): C, 62.46; H, 3.49; N, 17.34\%. Found: C, $62.61 ; \mathrm{H}, 3.39 ; \mathrm{N}, 17.22 \%$.

\subsection{Synthesis of N-(4-acetamidophenyl)-2-imino-2H-chromene-3-carboxamide (7)}

A suspension of equivalent ratio of $3(1.08 \mathrm{~g}, 5 \mathrm{mmol})$ and 2-hydroxybenzaldehyde $(0.60 \mathrm{~mL}, 5 \mathrm{mmol})$ was heated under reflux for $3 \mathrm{~h}$ in ethanol $(20 \mathrm{~mL})$ and five drops of piperidine. The resulting product was filtered, dried and recrystallized from EtOH/DMF (4:1) to yield 7.

Yellow crystals; yield (89\%); m.p. $249-250^{\circ} \mathrm{C}$. IR: $3292,3261(\mathrm{NH}), 1683(\mathrm{C}=\mathrm{O}) .{ }^{1} \mathrm{H}$ NMR $(\delta / \mathrm{ppm})$ : $2.08(\mathrm{~s}, 3 \mathrm{H}) 7.21-7.81(\mathrm{~m}, 8 \mathrm{H}), 8.31(\mathrm{~s}, 1 \mathrm{H}), 9.28(\mathrm{~s}, 1 \mathrm{H}), 10.18(\mathrm{~s}, 1 \mathrm{H}), 13.11(\mathrm{~s}, 1 \mathrm{H})$. MS (m/z, \%): 321 $\left(\mathrm{M}^{+}, 100\right), 254$ (34.0), 211 (27.4), 171 (88.2), 150 (74.2), 143 (65.1), 118 (32.3), 115 (33.3), 108 (87.6), 80 (34.2), 43 (18.6). Anal. Calcd. for $\mathrm{C}_{18} \mathrm{H}_{15} \mathrm{~N}_{3} \mathrm{O}_{3}$ (321.34): C, 67.28; H, 4.71; N, 13.08\%. Found: C, 67.11; H, $4.66 ; \mathrm{N}, 13.17 \%$.

\subsection{Synthesis of N-(4-acetamidophenyl)-2-cyano-2-(3-phenylthiazolylidene)acetamides 9} and 10

A suspension of scaffold $3(1.08 \mathrm{~g}, 5 \mathrm{mmol})$, phenyl isothiocyanate $(0.60 \mathrm{~mL}, 5 \mathrm{mmol})$ and solid $\mathrm{KOH}$ $(0.28 \mathrm{~g})$ was stirred in $20 \mathrm{~mL}$ DMF at room temperature for $8 \mathrm{~h}$. Stirring was continued for additional 4 $\mathrm{h}$ after in situ addition of the appropriate $\alpha$-halogenated reagents (ethyl chloroacetate, chloroacetone and/or phenacyl chloride) to the reaction mixture. The precipitate, which obtained after dilution with ice-cold water, was filtered and recrystallized.

N-(4-Acetamidophenyl)-2-cyano-2-(4-oxo-3-phenylthiazolidin-2-ylidene)acetamide (9)

Pale Brown powder; yield (72\%); m.p. 280-281 ${ }^{\circ} \mathrm{C}$ (from EtOH/DMF mixture). IR: 3408, $3302(\mathrm{NH})$, $2196(\mathrm{C} \equiv \mathrm{N}), 1743,1655(\mathrm{C}=\mathrm{O}) .{ }^{1} \mathrm{H} \operatorname{NMR}(\delta / \mathrm{ppm}): 2.01(\mathrm{~s}, 3 \mathrm{H}), 3.99(\mathrm{~s}, 2 \mathrm{H}), 7.40-7.55(\mathrm{~m}, 9 \mathrm{H}), 9.28$ (s, 1H), $9.84(\mathrm{~s}, 1 \mathrm{H})$. MS (m/z, \%): $392\left(\mathrm{M}^{+}, 54.0\right), 243$ (33.2), 215 (100.0), 150 (46.3), 132 (43.2), 108 
(34.5), 77 (50.0). Anal. Calcd. for $\mathrm{C}_{20} \mathrm{H}_{16} \mathrm{~N}_{4} \mathrm{O}_{3} \mathrm{~S}$ (392.43): C, 61.12; H, 4.11; N, 14.28\%. Found: C, 61.24; $\mathrm{H}, 4.15 ; \mathrm{N}, 14.15 \%$.

$\mathrm{N}$-(4-acetamidophenyl)-2-cyano-2-(4-methyl-3-phenylthiazol-2(3H)-ylidene)acetamide (10a):

Pale Brown powder; yield (78\%); m.p. $233-235^{\circ} \mathrm{C}$ (from EtOH/DMF mixture). IR: 3418, 3301 (NH), $2281(\mathrm{C} \equiv \mathrm{N}), 1662(\mathrm{C}=\mathrm{O}) .{ }^{1} \mathrm{H}$ NMR $(\delta / \mathrm{ppm}): 1.84(\mathrm{~s}, 3 \mathrm{H}), 2.01(\mathrm{~s}, 3 \mathrm{H}), 6.90(\mathrm{~s}, 1 \mathrm{H}), 7.30-7.60(\mathrm{~m}, 9 \mathrm{H})$, $8.75(\mathrm{~s}, 1 \mathrm{H}), 9.80(\mathrm{~s}, 1 \mathrm{H})$. MS (m/z, \%): $390\left(\mathrm{M}^{+}, 10.0\right), 217$ (63.5), 175 (79.9), 150 (10.6), 134 (34.0), 107 (100.0), 80 (21.7), 77 (10.6). Anal. Calcd. for $\mathrm{C}_{21} \mathrm{H}_{18} \mathrm{~N}_{4} \mathrm{O}_{2} \mathrm{~S}$ (390.46): C, 64.60; H, 4.65; N, $14.35 \%$. Found: C, 64.41; H, 5.54; N, 14.20\%.

N-(4-Acetamidophenyl)-2-cyano-2-(3,4-diphenylthiazol-2(3H)-ylidene)acetamide (10b):

Brown powder, yield (70\%); m.p. $264-265^{\circ} \mathrm{C}$ (from EtOH). IR: 3391, $3295(\mathrm{NH}), 2172(\mathrm{C} \equiv \mathrm{N}), 1661$ $(\mathrm{C}=\mathrm{O}) .{ }^{1} \mathrm{H}$ NMR $(\delta / \mathrm{ppm}): 2.07(\mathrm{~s}, 3 \mathrm{H}), 7.19-7.57(\mathrm{~m}, 14 \mathrm{H}), 7.88(\mathrm{~s}, 1 \mathrm{H}), 9.81(\mathrm{~s}, 1 \mathrm{H}), 9.86(\mathrm{~s}, 1 \mathrm{H})$. Anal. Calcd. for $\mathrm{C}_{26} \mathrm{H}_{20} \mathrm{~N}_{4} \mathrm{O}_{2} \mathrm{~S}$ (452.53): C, 69.01; H, 4.45; N, 12.38\%. Found: C, 68.13; H, 4.37; N, $12.26 \%$.

\subsection{Synthesis of N-(4-acetamidophenyl)-2-cyano-3-mercapto-3-(phenylamino)acrylamide}

A suspension of scaffold 3 (1.08 g, $5 \mathrm{mmol})$, phenyl isothiocyanate $(0.60 \mathrm{~mL}, 5 \mathrm{mmol})$ and solid $\mathrm{KOH}$ $(0.28 \mathrm{~g})$ was stirred in $20 \mathrm{~mL}$ DMF. After stirring for $8 \mathrm{~h}$, the mixture was decanted into ice-cold water and neutralized with dilute acid. The precipitate that obtained was separated by filtration and recrystallized from ethanol.

Orange powder; yield $(75 \%)$, m.p. $139-140^{\circ} \mathrm{C}$. IR: $3457,3318(\mathrm{NH}), 2216(\mathrm{C} \equiv \mathrm{N}), 1665(\mathrm{C}=\mathrm{O}) .{ }^{1} \mathrm{H}$ NMR $(\delta / \mathrm{ppm}): 2.07(\mathrm{~s}, 3 \mathrm{H}), 7.46-7.71(\mathrm{~m}, 10 \mathrm{H}), 9.85(\mathrm{~s}, 1 \mathrm{H}), 10.17(\mathrm{~s}, 1 \mathrm{H})$. Anal. Calcd. for $\mathrm{C}_{18} \mathrm{H}_{16} \mathrm{~N}_{4} \mathrm{O}_{2} \mathrm{~S}$ (352.41): C, 61.35; H, 4.58; N, 15.90\%. Found: C, 61.51; H, 4.65; N, $15.81 \%$.

\subsection{Synthesis of N-(4-acetamidophenyl)-4-amino-2-(phenylamino)-5-} substitutedthiophene-3-carboxamides 12

A suspension of thiocarbamoyl scaffold 11 (0.70 g, $2 \mathrm{mmol})$ and the appropriate chloroketone $(2 \mathrm{mmol})$ was refluxed for $4 \mathrm{~h}$ in EtOH $(20 \mathrm{~mL})$ and five drops of $\mathrm{Et}_{3} \mathrm{~N}$. The precipitate that formed on cooling to room temperature was separated by filtration and dried to furnish the target thiophene scaffolds.

$\mathrm{N}$-(4-Acetamidophenyl)-5-acetyl-4-amino-2-(phenylamino)thiophene-3-carboxamide (12a):

Brown powder; yield (65\%), m.p. $188-190^{\circ} \mathrm{C}$. IR: 3343, $3300\left(\mathrm{NH}_{2}\right.$ and $\left.\mathrm{NH}\right), 1657(\mathrm{C}=\mathrm{O}) .{ }^{1} \mathrm{H}$ NMR $(\delta / \mathrm{ppm}): 2.01(\mathrm{~s}, 3 \mathrm{H}), 2.32(\mathrm{~s}, 3 \mathrm{H}), 7.04-7.52(\mathrm{~m}, 10 \mathrm{H}), 9.88(\mathrm{~d}, 2 \mathrm{H}), 11.55(\mathrm{~s}, 1 \mathrm{H}), 11.88(\mathrm{~s}, 1 \mathrm{H}) . \mathrm{MS}$ (m/z, \%): $408\left(\mathrm{M}^{+}, 2.29\right), 392$ (26.9), 241 (40.2), 232 (12.3), 217 (48.0), 187 (20.1), 175 (70.8), 150 (50.2), 134 (44.7), 107 (100.0), 80 (9.6), 77 (37.2), 51 (13.9). Anal. Calcd. for $\mathrm{C}_{21} \mathrm{H}_{20} \mathrm{~N}_{4} \mathrm{O}_{3} \mathrm{~S}$ (408.48): C, 61.75; H, 4.94; N, 13.72\%. Found: C, 61.54; H, 4.98; N, 13.61\%.

$\mathrm{N}$-(4-acetamidophenyl)-4-amino-5-benzoyl-2-(phenylamino)thiophene-3-carboxamide (12b)

Pale brown powder; yield $(80 \%)$, m.p. $275-276^{\circ} \mathrm{C}$ (from ethanol). IR: 3383, 3293, $\left(\mathrm{NH}_{2}\right.$ and $\left.\mathrm{NH}\right), 1659$ $(\mathrm{C}=\mathrm{O}) .{ }^{1} \mathrm{H}$ NMR $(\delta / \mathrm{ppm}): 2.07(\mathrm{~s}, 3 \mathrm{H}), 7.29-7.87(\mathrm{~m}, 15 \mathrm{H}),, 9.91(\mathrm{~d}, 2 \mathrm{H}), 10.87(\mathrm{~s}, 1 \mathrm{H}), 11.44(\mathrm{~s}, 1 \mathrm{H})$. MS (m/z, \%): $470\left(\mathrm{M}^{+}, 3.4\right), 392$ (57.6), 319 (12.0), 294 (18.0), 243 (34.8), 215 (100.0), 150 (50.0), 132 (38.0), 108 (28.5), 77 (57.1), 51 (8.9). Anal. Calcd. for $\mathrm{C}_{26} \mathrm{H}_{22} \mathrm{~N}_{4} \mathrm{O}_{3} \mathrm{~S}$ (470.55): C, 66.37; H, 4.71; N, $11.91 \%$. Found: C, 66.49; H, 4.77; N, $11.82 \%$.

\subsection{Synthesis of N-(4-acetamidophenyl)-2-cyano-3-(methylthio)-3-(phenylamino)- acrylamide (13)}

A suspension of scaffold 3 (1.08 g, $5 \mathrm{mmol})$, phenyl isothiocyanate $(0.60 \mathrm{~mL}, 5 \mathrm{mmol})$ and solid $\mathrm{KOH}$ $(0.28 \mathrm{~g})$ was stirred in $20 \mathrm{~mL}$ DMF. After stirring for $8 \mathrm{~h}$, methyl iodide $(0.35 \mathrm{~mL}, 5 \mathrm{mmol})$ was decanted to the reaction suspension and stirring was continued for additional $4 \mathrm{hr}$. The precipitate, which obtained after dilution with ice-cold water, was picked up filtration and recrystallized from ethanol.

Pale yellow powder, yield $(80 \%)$, m.p. $207-208^{\circ} \mathrm{C}$. IR: $3406,3303,3156(\mathrm{NH}), 2198(\mathrm{C} \equiv \mathrm{N}), 1675$ $(\mathrm{C}=\mathrm{O}) .{ }^{1} \mathrm{H}$ NMR $(\delta / \mathrm{ppm}): 2.01(\mathrm{~s}, 3 \mathrm{H}), 2.23(\mathrm{~s}, 3 \mathrm{H}), 7.24-7.49(\mathrm{~m}, 9 \mathrm{H}), 9.45(\mathrm{~s}, 1 \mathrm{H}), 9.84(\mathrm{~s}, 1 \mathrm{H}), 11.76$ 
(s, 1H). Anal. Calcd. for $\mathrm{C}_{19} \mathrm{H}_{18} \mathrm{~N}_{4} \mathrm{O}_{2} \mathrm{~S}$ (366.44): C, 62.28; H, 4.95; N, 15.29\%. Found: C, 62.17; H, 4.87; N, $15.12 \%$.

\subsection{Synthesis of $N$-(4-Acetamidophenyl)-5-amino-3-(phenylamino)-1 H-pyrazole-4- carboxamide (14)}

A mixture of 13 (0.73 g, $2 \mathrm{mmol})$ and hydrazine hydrate $(0.20 \mathrm{ml}, 5 \mathrm{mmol})$ was refluxed for $4 \mathrm{~h} \mathrm{in} 20 \mathrm{ml}$ EtOH. The precipitate that formed on cooling to room temperature was picked up by filtration and dried to furnish the target pyrazole scaffold.

Pale yellow powder, yield (60\%), m.p. $184-185^{\circ} \mathrm{C}(\mathrm{EtOH})$. IR: 3445, 3261, $3176\left(\mathrm{NH}_{2}\right.$ and NH), 1651 $(\mathrm{C}=\mathrm{O}) .{ }^{1} \mathrm{H}$ NMR $(\delta / \mathrm{ppm}): 2.00(\mathrm{~s}, 3 \mathrm{H}), 6.05(\mathrm{~s}, 2 \mathrm{H}), 6.25(\mathrm{~s}, 2 \mathrm{H}), 6.78(\mathrm{t}, 1 \mathrm{H}), 7.18-7.27(\mathrm{~m}, 4 \mathrm{H}), 7.45$ $(\mathrm{d}, 2 \mathrm{H}), 7.55(\mathrm{~d}, 2 \mathrm{H}, \mathrm{J}=7.8 \mathrm{~Hz}), 8.59(\mathrm{~s}, 1 \mathrm{H}), 8.78(\mathrm{~s}, 1 \mathrm{H})$. Anal. Calcd. for $\mathrm{C}_{18} \mathrm{H}_{18} \mathrm{~N}_{6} \mathrm{O}_{2}(350.38): \mathrm{C}$, $61.70 ; \mathrm{H}, 5.18 ; \mathrm{N}, 23.99 \%$. Found: C, $61.59 ; \mathrm{H}, 5.25 ; \mathrm{N}, 23.86 \%$.

\subsection{In Vitro Antitumor Activity}

Mammary gland breast cancer (MCF-7) cell line was obtained from ATCC via Holding company for biological products and vaccines (VACSERA), Cairo, Egypt.

MTT assay [37-39]: The cell lines were employed to reflect the inhibitory effects of compounds on cell growth using the MTT assay. This assay is depended on the change of the yellow 3-(4,5-dimethylthiazol2-yl)-2,5-diphenyltetrazolium bromide (MTT) to a purple formazan derivative containing mitochondrial succinate dehydrogenase in viable cells [37-39].

\section{Conclusion}

The present study has been focused on the synthesis and investigation the anticancer activity of new thiazole, pyridinone, thiophene, chromene and pyrazole derivatives containing biologically active acetanilide nucleus. Some of the newly synthesized compounds 4c, 5f, 9, 10a and 10b exhibited significant activity (very strong) compared to the control drug, 5-fluorouracil. Compounds 3, 4a 4b, 6c and $12 \mathrm{~b}$ are strong cytotoxic drugs. Compounds 5c,5d,6a and $12 \mathrm{a}$ exhibited a moderate activity and compounds 5b, 6b, 6d, 7 and 13 showed a weak activity, while compound $5 \mathrm{a}$ is inactive cytotoxic drug.

\section{References}

1. El-Gaml, K. M. (2015) Synthesis and antimicrobial evaluation of new polyfunctionally substituted heterocyclic compounds derived from 2-cyano-N-(3-cyanoquinolin-2-yl)acetamide. American Journal of Organic Chemistry, 5, $1-9$.

2. Darwish, E. S., Fattah, A. M. A., Attaby, F. A. and Al-Shayea, O. N. (2014) Synthesis and antimicrobial evaluation of some novel thiazole, pyridone, pyrazole, chromene, hydrazone derivatives bearing a biologically active sulfonamide moiety. International Journal of Molecular Sciences, 15, 1237-1254.

3. Panchal, A. D., Kunjadia, P. D. and Patel, P. M. (2011) Synthesis and biological evaluation of chalcone derivatives linked triazoles. International Journal of Pharmaceutical Sciences and Drug Research, 3, 331-337.

4. Alafeefy, A. M., Isik, S., Abdel-Aziz, H. A., Ashour, A. E., Vullo, D., Al-Jaber, N. A. and Supuran, C. T. (2013) Carbonic anhydrase inhibitors: Benzenesulfonamides incorporating cyanoacrylamide moieties are low nanomolar/subnanomolar inhibitors of the tumor-associated isoforms IX and XII. Bioorganic and Medicinal Chemistry, 21, 1396-1403.

5. Arora, M., Saravanan, J., Mohan, S. and Bhattacharjee, S. (2013) Synthesis, characterization and antimicrobial activity of some Schiff bases of 2-amino-N-(p-acetamidophenyl carboxamido)-4,5,6,7-tetramethylene thiophenes. International Journal of Pharmacy and Pharmaceutical Sciences, 5, 315-319.

6. Khokhani, K., Khatri, T. and Patel, P. (2013). One Pot Synthesis of bioactive novel cyanopyridones. Journal of the Korean Chemical Society, 57, 476-482.

7. Fadda, A. A., Mukhtar, M. M. and Refat, H. M. (2012) Utility of activated nitriles in the synthesis of some new heterocyclic compounds. American Journal of Organic Chemistry, 2, 32-40. 
8. Al-Saadi, M. S., Faidallah, H. M. and Rostom, S. A. F. (2008) Synthesis and biological evaluation of some 2,4,5trisubstituted thiazole derivatives as potential antimicrobial and anticancer agents. Archiv der Pharmazie Chemistry in Life Sciences, 341, 424-434.

9. Bondock, S., Rabie, R., Etman, H. A. and Fadda, A. A. (2008) Synthesis and antimicrobial activity of some new heterocycles incorporating antipyrine moiety. European Journal of Medicinal Chemistry, 43, 2122-2129.

10. Bondock, S., Khalifa, W. and Fadda, A. A. (2007) Synthesis and antimicrobial evaluation of some new thiazole, thiazolidinone and thiazoline derivatives starting from 1-chloro-3,4-dihydronaphthalene-2-carboxaldehyde. European Journal of Medicinal Chemistry, 42, 948-954.

11. Karegoudar, P., Karthikeyan, M. S., Prasad, D. J., Mahalinga, M., Holla, B. S. and Kumari, N. S. (2008) Synthesis of some novel 2,4-disubstituted thiazoles as possible antimicrobial agents. European Journal of Medicinal Chemistry, 43, 261-267.

12. Chandanshive, J. Z., Pedro, B. G., Tiznado, W., Bonini, B. F., Caballero, J. and Femoni, C. (2012) 1,3-Dipolar cycloaddition of nitrile imines with $\alpha, \beta$-unsaturated lactones, thiolactones and lactams: Synthesis of ring-fused pyrazoles. Tetrahedron, 68, 3319-3328.

13. Bekhit, A. A. and Aziem T. A. (2004) Design, synthesis and biological evaluation of some pyrazole derivatives as anti-inflammatory-antimicrobial agents. Bioorganic and Medicinal Chemistry, 12, 1935-1945.

14. El-Emary, T. and El-Mohsen, S. (2012) Multi-component one-pot synthesis and antimicrobial activities of 3methyl-1,4-diphenyl-7-thioxo-4,6,8,9-tetrahydro-pyrazolo[5,4-b]pyrimidino[5,4-e]pyridine-5-one and related derivatives. Molecules, 17, 14464-14483.

15. Abu-Hashem, A. A., Gouda, M. A. and Badria, F. A. (2010) Synthesis of some new pyrimido $\left[2^{\prime}, 1^{\prime}: 2,3\right]$ thiazolo[4,5-b]quinoxaline derivatives as anti-inflammatory and analgesic agents. European Journal of Medicinal Chemistry, 45, 1976-1981.

16. Gouda, M. A., Berghot, M. A., Abd El-Ghani, G. E. and Khalil, A. M. (2010). Synthesis and antimicrobial activities of some new thiazole and pyrazole derivatives based on 4,5,6,7-tetrahydrobenzothiophene moiety. European Journal of Medicinal Chemistry, 45, 1338-1345.

17. Khalil, A. M., Berghot, M. A. and Gouda M. A. (2009) Synthesis and antibacterial activity of some new thiazole and thiophene derivatives. European Journal of Medicinal Chemistry, 44, 4434-4440.

18. Gouda, M. A., Berghot, M. A., Shoeib, A. I. and Khalil, A. M. (2010) Synthesis and antimicrobial of certain new thiazolidinone, thiazoline and thiophene derivatives. Phosphorus, Sulfur, and Silicon and the Related Elements, 185, 1455-1462.

19. Gouda, M. A. and Abu-Hashem A. A. (2011) Synthesis, characterization, antioxidant and antitumor evaluation of some new thiazolidine and thiazolidinone derivatives. Archiv der Pharmazie - Chemistry in Life Sciences, 11, $170-177$.

20. Wilby, M. J. and Hutchinson P. J. (2004) The pharmacology of chlormethiazole: A potential neuroprotective agent. CNS Drug Reviews, 10, 281-294.

21. Harnett, J. J., Roubert, V., Dolo, C., Charnet, C., Spinnewyn, B., Cornet, S., Rolland, A., Marin, J. G., Bigg, D. and Chabrier, P. E. (2004) Phenolic thiazoles as novel orally-active neuroprotective agents. Bioorganic and Medicinal Chemistry Letters, 14, 157-160.

22. Lesyk, R., Zimenkovsky, B., Atamanyuk, D., Jensen, F., Kononowicz, K. K. and Gzella, A. (2006) Anticancer thiopyrano[2,3-d][1,3] thiazol-2-ones with norbornane moiety. Synthesis, cytotoxicity, physico-chemical properties, and computational studies. Bioorganic and Medicinal Chemistry, 14, 5230-5240.

23. Kashfi, K. (2009) Anti-inflammatory agents as cancer therapeutics. Advances in pharmacology, 57, 31-89.

24. Modiya, P. R. and Patel, C. N. (2012) Synthesis and screening of antibacterial and antifungal activity of 5chloro-1,3-benzoxazol-2(3H)-one derivatives. Organic and Medicinal Chemistry Letters, 2, 29.

25. Al-Said, M. S., Ghorab, M. M. and Nissan, Y. M. (2012) Dapson in heterocyclic chemistry, part VIII: Synthesis, molecular docking and anticancer activity of some novel sulfonylbiscompounds carrying biologically active 1,3dihydropyridine, chromene and chromenopyridine moieties. Chemistry Central Journal, 6, 64.

26. Dragovich, P. S., Prins, T. J., Zhou, R., Brown, E. L., Maldonado, F. C., Fuhrman, S. A., Zalman, L. S., Tuntland, T., Lee, C. A. and Patick, A. K. (2002) Structure-based design, synthesis, and biological evaluation of irreversible human rhinovirus 3C protease inhibitors. Part 6: Structure-activity studies of orally bioavailable, 2pyridone-containing peptidomimetics. Journal of Medicinal. Chemistry, 45, 1607-1623. 
27. Dragovich, P. S., Prins, T. J. Zhou, R., Johnson, T. O., Brown, E. L., Maldonado, F. C., Fuhrman, S. A., Zalman, L. S., Patick, A. K. and Matthews, D. A. (2002) Structure-based design, synthesis, and biological evaluation of irreversible human rhinovirus 3C protease inhibitors. Part 7: Structure-activity studies of bicyclic 2-pyridone-containing peptidomimetics. Bioorganic and Medicinal Chemistry Letters, 12, 733-738.

28. Ye, D., Zhang, Y., Wang, F., Zheng, M., Zhang, X., Luo, X., Shen, X., Jiang, H. and Liu, H. (2010) Novel thiophene derivatives as PTP1B inhibitors with selectivity and cellular activity. Bioorganic and Medicinal Chemistry, 18, 1773-1782.

29. Kulandasamy, R., Adhikari, A. V. and Stables, J. P. (2009) A new class of anticonvulsants possessing $6 \mathrm{~Hz}$ activity: 3,4-Dialkyloxy thiophene bishydrazones. European Journal of Medicinal Chemistry, 44, 4376-4384.

30. Khalil, A. M., Berghot, M. A., Ghada E. A. and Gouda, M. A. (2010), Synthesis and antimicrobial evaluation of some new thiophene derivatives. European Journal of Medicinal Chemistry, 40, 1658-1669.

31. Ronad, P. M., Noolvi, M. N., Sapkal, S., Dharbhamulla, S. and Maddi, V. S. (2010) Synthesis and antimicrobial activity of 7-(2-substitutedphenylthiazolidinyl)-benzopyran-2-one derivatives. European Journal of Medicinal Chemistry, 45, 85-89.

32. Borges, F., Roleir, F., Santana, L. and Uriare E. (2005) Simple coumarins and analogues in medicinal chemistry: occurrence, synthesis and biological activity. Current Medicinal Chemistry, 12, 887-916.

33. Fadda, A. A., Berghot, M. A., Amer, F. A., Badway, D. S. and Bayoumy, N. M. (2012) Synthesis and antioxidant and antitumor activity of novel pyridine, chromine, thiophene and thiazoles derivatives. Archiv der Pharmazie, 435, 378-385.

34. Fadda, A. A. and Youssif, E. H. E. (2011) Synthesis of some new chromene derivatives. Synthetic Communications, 41, 677-694.

35. El-Shafei, A., Fadda, A. A., Abdel-Gawad, I. I. and Youssif, E. H. E. (2009) Stereospecificity of Diels-Alder reactions validated using ab initio calculations: Synthesis of novel coumarin and phenanthridine derivatives. Synthetic Communications, 39, 2954-2972.

36. Lahtchev, K. L. Batovska, D. I., Parushev, St. P., Ubiyvovk, V. M. and Sibirny, A. A. (2008) Antifungal activity of chalcones: A mechanistic study using various yeast strains. European Journal of Medicinal Chemistry, 43, 2220-2228.

37. Tim, M. (1983). Rapid colorimetric assay for cellular growth and survival: Application to proliferation and cytotoxicity assays. Journal of Immunological Methods, 65, 55-63.

38. Francois, D. and Rita, L. (1986) Rapid colorimetric assay for cell growth and survival: Modifications to the tetrazolium dye procedure giving improved sensitivity and reliability. Journal of Immunological Methods, 89, 271-277.

39. Helena, J. M., Nader, N. H., Micheal, A. B., David, H. G., Mary, J. S., Kerri, A. S., Kevin, B., Ruth, H., Stephen, G., Mohanraj, D., Gerald, A. S., Vikas, P. S., Donald, W. K. and Ralph, R. W. (1998). Combined effects of angiostatin and ionizing radiation in antitumour therapy. Nature, 394, 287-291. 